\title{
Aplicability of 2D and 3D isovists and visibility graph analysis for evaluating urban vulnerability to crime: the case of Boa Viagem, in Recife
}

\section{SIGRADI2018 TECHNOPOLITICAS \\ xxii congresso da sociedade iberoamericana de gráfica digital 22th conference of the iberoamerican society of digital graphics 07|08|09|novembro|2018 iau usp | são carlos | sp br}

\author{
Silvio Pereira Bezerra de Melo Junior \\ Universidade Católica de Pernambuco | Brazil | silviomeloj@gmail.com \\ Robson Canuto da Silva \\ Universidade Católica de Pernambuco | Brazil | robsoncanuto.arq@gmail.com
}

\begin{abstract}
This work aims to investigate the applicability of 2D and 3D isovists, as well as Visibility Graph Analysis (VGA), for evaluating urban vulnerability to crime. The methodology is based on correlations between number of crime occurrences and measurements of 2D and 3D isovists, and mean values of visual integration (VGA). The 2D isovists were produced through DeCoding Spaces Toolbox for Grasshopper and the 3D isovists were generated by using algorithms within Rhinoceros and Grasshopper. VGA maps were elaborated within DepthmapX. For this study, were selected nine street segments of Boa Viagem, located in Recife-PE, a neighbourhood which is known for high rates of robberies. Although the number of samples is reduced, the results suggest that criminals prefer much more visually integrated spaces with low occlusivity and fewer spatial cavities.
\end{abstract}

\section{INTRODUÇÃO}

O trabalho investiga relações entre visibilidade e criminalidade em espaços públicos do Recife.

Visibilidade é, etimologicamente, a propriedade pela qual os corpos são percebidos no espaço (Michaelis, 2016). Todavia, a visibilidade de que trata este estudo está intrinsecamente relacionada à noção de isovista - o campo visível a partir de um determinado ponto no espaço, considerando as barreiras que o limitam (Benedikt, 1979). Esse conceito foi introduzido por Michael Benedikt, em 1979, na revista Environment and Planning, sendo considerado um dos primeiros métodos científicos a correlacionar espaço e visibilidade. Nos anos de 1990 e início dos anos 2000, com os avanços no desenvolvimento de ferramentas computacionais de análise espacial, a noção de isovista é retomada por Alasdair Turner e seus colegas da University College London. Turner et al (2001) desenvolveram a Visibility Graph Analysis (VGA), expandido a noção de isovista, passando a considerar não apenas a visibilidade de um ponto, mas de todos os pontos contidos no espaço.

Criminalidade, por outro lado, vem de crime, ou seja, transgressão da lei, violação das regras que a sociedade considera como indispensáveis à sua existência e organização. O Código Penal Brasileiro (Brasil, 1940) classifica o crime em onze categorias: (1) contra a pessoa, (2) contra o patrimônio, (3) contra a propriedade imaterial, (4) contra a organização do trabalho, (5) contra o sentimento religioso e o respeito aos mortos, (6) contra os costumes, (7) contra a família, (8) contra a incolumidade pública, (9) contra a paz pública, (10) contra a fé pública e (11) contra a administração pública. Em particular, os crimes contra o patrimônio, isto é, os roubos e furtos, implicam ocorrências em um determinado ponto do espaço.
Assim sendo, visibilidade, criminalidade e espaço estão intrinsecamente relacionados, mas são aspectos cujas inter-relações são ainda pouco investigadas, o que é constatado por meio de breve revisão de literatura. Em geral, estudos relativos a aspectos de configuração espacial que facilitam ou restringem a ocorrência de crimes no meio urbano têm abordado fatores como uso, interface, constituições, densidade, integração, tipologia, permeabilidade física e visual, além da proporção entre espaços público e privado. Todavia, a visibilidade tem sido pouco abordada, o que motiva o desenvolvimento de mais estudos neste campo. Ainda que haja um interesse cada vez maior sobre o tema e que estudos sobre morfologia urbana e criminalidade remontem à segunda metade do século $X X$, pouco se conhece sobre o modo como o criminoso enxerga a cidade e tira partido dela para praticar 0 delito e sobre em que medida a configuração espacial da cidade promove ou restringe a criminalidade. Entende-se que, os métodos de isovistas, isovistas 3D e Visibility Graph Analysis (VGA) podem auxiliar a responder algumas dessas questões, aspecto pelo qual o trabalho discute a aplicabilidade desses instrumentos na avaliação de vulnerabilidade urbana ao crime, tendo como caso o bairro de Boa Viagem, em Recife.

\section{O BAIRRO DE BOA VIAGEM COMO OBJETO URBANO}

O bairro de Boa Viagem está localizado na Zona Sul do Recife, cidade que tem registrado altas taxas de criminalidade, uma das maiores do país (Pernambuco, 2011). Como forma de proteção, observa-se na arquitetura da cidade a emergência de soluções como gradis, muros altos, cercas elétricas e câmeras de vigilância, convertendo os espaços privados em enclaves fortificados e os espaços públicos em locais inseguros. Essas transformações ficaram bastante evidentes em bairros de classe média e alta como Boa Viagem. 
Contudo, em Boa Viagem, o que chama a atenção são transformações ainda mais significativas ocorridas a partir da segunda metade do século $X X$ tanto nas formas edilícias como nas relações estabelecidas entre essas formas e os espaços públicos. Até os anos de 1950, o bairro era conformado predominantemente por casas de veraneio, com acessibilidade direta à rua ou a espaços de transição (jardins e terraços privativos). A partir dos anos de 1950, são implantados os primeiros edifícios em altura, como o Edifício Califórnia (1956) (Figura 1A) projetado do arquiteto Acácio Gil Borsoi e os edifícios Holiday (1957) (Figura 1B) e Acaiaca (1958) (Figura 1C) projetados por Delfim Amorim. Esses edifícios ainda preservavam relações estreitas entre os espaços públicos e privados, como acessibilidade direta à rua, faces transparentes, uso comercial no pavimento térreo e muros baixos. A partir dos anos de 1980, Boa Viagem se torna um território verticalmente adensado após sucessivas leis urbanísticas cujos parâmetros determinaram sua configuração atual (Figura 1D, 1F e 1E). O território que inicialmente contava com casas e edifícios mais próximos à rua, converteu-se num espaço conformado por condomínios verticais fechados com muros cegos. Sobretudo no térreo, edifícios perderam o que Jane Jacobs chama de os "olhos da rua" (Jacobs, 2011, p. 35) e ganharam circuitos fechados de TV, que prometem a "segurança" dos proprietários.

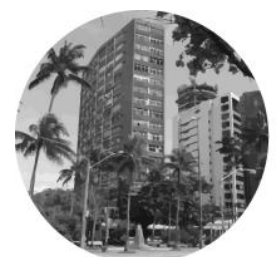

[A]

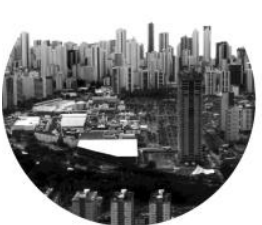

[D]

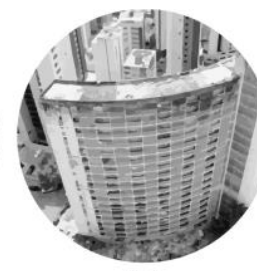

[B]

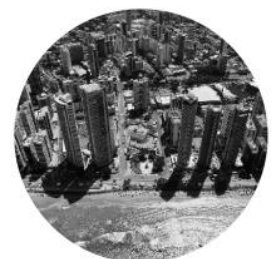

[E]

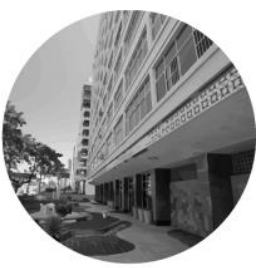

[C]

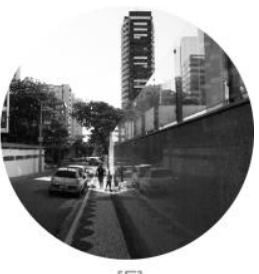

[F]
Figura 1 - Edifício Califórnia $[A]$, Holiday $[B]$, Acaiaca [C], fotos aéreas [D e E], interface de um edifício contemporâneo com o espaço público [F]. Fonte: autores.

Estabelece-se, assim, uma relação de causa e efeito, uma vez que essas mudanças ao mesmo tempo em que salvaguardam o espaço privado, promovem a insegurança no domínio público, potencializando a ocorrência de crimes. A rígida relação entre o âmbito público e privado está, possivelmente, gerando oportunidades para os assaltantes, uma vez que se verifica um aumento de $18,4 \%$ no número de roubos e furtos ocorridos entre 2015 e 2016 . O papel da interface das edificações na vulnerabilidade do espaço para a ocorrência de crimes é um aspecto extensivamente investigado por Monteiro e Cavalcanti (2015).

A interface, contudo, não deve ser exclusivamente a razão para o aumento da vulnerabilidade ao crime em Boa Viagem, ao menos do ponto de vista morfológico. A Discussões sobre relações entre visibilidade (campo visível do espaço) e criminalidade no meio urbano ainda são uma lacuna, tanto nos estudos locais (sobre Boa Viagem) como nos estudos internacionais, o que é verificado por meio de breve revisão de literatura (textos clássicos e contemporâneos).

\section{REVISÃO DE LITERATURA}

\section{A CARÊNCIA DE ESTUDOS SOBRE O PAPEL DA VISIBILIDADE NA OCORRÊNCIA DE CRIMES}

É cada vez maior o número de investigações que buscam compreender o papel da configuração espacial das cidades na ocorrência de crimes. Estudos desta natureza remontam aos anos de 1960 e 1970, a partir de publicações de Jane Jacobs e Oscar Newman. Com o avanço dos métodos computacionais de análise espacial, ocorrido entre os anos de 1980 e 2000, o número de pesquisas neste campo aumentou consideravelmente, permitindo um maior conhecimento de atributos do espaço urbano que contribuem para a ocorrência de crimes. Esses estudos têm predominantemente abordado grande diversidade de atributos (uso, interface, constituições, densidade, integração, tipologia, permeabilidade física, permeabilidade visual e proporção entre espaços público e privado), mas a visibilidade é pouco investigada.

Em The Death and Life of Great American Cities, Jane Jacobs abordou a influência da morfologia urbana na emergência de espaços urbanos mais seguros, o que seria garantido pela clara definição da interface entre espaços públicos e privados, pelo estimulo à variedade de usos e aumento do número de usuários do espaço público (ou aumento da densidade populacional). Jacobs (1961) defendia principalmente a ideia de considerar as pessoas anônimas como garantia de segurança e não como fonte de perigo. Nesta concepção, a presença de usuários no espaço público (conhecidos ou desconhecidos) contribuiria para maior vigilância social do espaço urbano e, consequentemente, para a segurança urbana.

Em contraposição às ideias de Jacobs (1961), Newman (1972) argumentava que, pessoas anônimas eram potenciais fontes de perigo e defendia a reestruturação física de layouts urbanos, visando maior controle de acessos como estratégia para a promoção de segurança urbana. Em Creating Defensible Space, Newman (1996) argumentava que, o espaço urbano deveria ser segmentado em mini-neighborhoods, com acesso controlado por portões, diminuindo a permeabilidade física. Além disto, defendia a ideia de que a baixa densidade, a baixa variedade de usos e a maximização do espaço privado eram aspectos que tornavam os espaços públicos mais seguros.

Desenvolvido com base nas ideias do manual Crime Prevention Through Environmental Design (CPTED), Safescape (Zelinka e Brennan, 2000) defendia a importância da construção de um senso de comunidade como forma de redução da criminalidade urbana e do medo de crimes no meio urbano. Zelinka e Brennan (2000) argumentavam que, através do desenho urbano é possível reduzir as oportunidades para a ação de assaltantes. Nesta concepção, a segurança das áreas públicas é assegurada ao se estimular a cidade aberta, permeável, densamente habitada, com alta variedade de usos, áreas públicas e maior número de acessos (constituições) de modo a promover a vigilância social. 
Nos anos 2000, surgiram diversos estudos que abordaram a questão da criminalidade no meio urbano sob o ponto de vista da sintaxe do espaço (Hillier, 2004; Hillier e Sahbaz, 2008). Em Can streets be made safe?, Hillier (2004) defendeu que, a resposta para a indagação título de seu artigo é a pesquisa através da sintaxe espacial. Hillier (2004) comparou os estudos precedentes de Jacobs (1961) e Newman (1972) e argumentou que, o uso indiscriminado dos conceitos propostos por Oscar Newman tem sido responsável pela criação de espaços fragmentados, subutilizados e com baixa visibilidade, apontando a consideração de dois aspectos sintáticos cruciais para o entendimento das relações entre espaço e crime: a visibilidade e a integração. Em estudo correlato - An evidence base approach to crime and urban design: Or, can we have vitality, sustainability and security all at once? -, Hillier e Sahbaz (2008) sugerem que, as soluções para instituir espaços urbanos seguros têm se dividido em dois tipos de tentativa: espaços fechados (enclausuramento como forma de proteção) e espaços abertos (permeabilidade, acessibilidade e visibilidade como forma de segurança). Hillier e Sahbaz (2008) argumentam que, características espaciais diferentes sugerem a ocorrência de crimes também diferentes: lugares com alto nível de copresença favorecem a ação de pequenos furtos, enquanto espaços mais vazios e pouco observados estimulam ações mais demoradas (roubo de veículos, casas, etc.).

Em Spatial profile of urban segments: Assessing place vulnerability to crime, Monteiro e Cavalcanti (2015) apresentam uma perspectiva diferente para o campo de estudo, pois investigam a possibilidade de avaliar a vulnerabilidade do espaço urbano público à criminalidade, tendo como objeto o caso de Boa Viagem. Para avaliação do grau de vulnerabilidade o estudo estabeleceu fatores classificados como diretamente proporcionais à segurança, o que significa dizer que quanto maior seu valor numérico, menos vulnerável ao crime é o espaço. Tal classificação está dividida em aspectos qualitativos e quantitativos. Os qualitativos são variedade de usos (diversidade de atividades), interface urbana (o que as autoras consideram como sendo o nível de transparência apenas), número de constituições (acessos diretos entre espaço público e privado) e densidade populacional. Os aspectos quantitativos dizem respeito a medidas sintáticas de integração global e integração local. Ambos são analisados a partir de segmentos urbanos (faces de quadra), juntamente com os dados de ocorrência de crimes contra o patrimônio (roubos e furtos) coletados entre 2010 e 2012. Concluiu-se que, onde o nível de integração local e o comprimento do segmento são maiores, o número de crimes no segmento é mais elevado, o que é justificado pelo fato de as pessoas terem seu comportamento observado durante maior período em quadras grandes, dando tempo de ação para o criminoso (Monteiro e Cavalcanti, 2015).

Em síntese, a literatura tem sido centrada na contradição entre permeabilidade e defensibilidade do espaço como estratégia de prevenção ao crime (Jacobs, 1961; Newman, 1972; Zelinka e Brennan, 2000), bem como na investigação de atributos qualitativos e quantitativos do espaço que têm reflexos na ocorrência de crimes, visando aferi-los e implementá-los como forma de prevenção (Hillier, 2004; Hillier e Sahbaz, 2008; Monteiro, 2015). Apesar da diversidade de aspectos analisados por estes estudos, a visibilidade é pouco explorada. Mesmo na abordagem de Hillier (2004), a visibilidade e a intervisibilidade são tradadas de modo qualitativo, concluindo que, espaços com maior nível de visibilidade são mais utilizados pelos usuários e, por conseguinte, mais seguros. No entanto, observa-se que, a dimensão da visibilidade tem sido raramente explorada do ponto de vista quantitativo nas questões de segurança e criminalidade, o que motiva 0 desenvolvimento de estudos neste campo.

\section{AVANÇOS RECENTES NOS ESTUDOS SOBRE O CAMPO VISÍVEL DO ESPAÇO}

O espaço e tudo que o delimita compõem um arranjo de natureza complexa que pode ser percebido pelo observador. A "capacidade de perceber o espaço pelo sentido da visão" tem sido definida como visibilidade (Michaelis, 2016). Visibilidade, portanto, pressupõe um sujeito e um objeto (ou um limite), ainda que esteja muito distante do nosso campo de visão. No âmbito do espaço arquitetônico, paredes, portas e, muitas vezes, mobílias atuam como elementos que delimitam o campo de visão, enquanto no espaço urbano são edificações, muros, portões, dentre outros, os elementos construídos que cumprem tal função. Ao construirmos paredes e edifícios, moldamos um determinado campo visual - uma forma espacial ou um sistema de barreiras visuais que facilita ou restringe nossa visão e, consequentemente, as possibilidades de interação visual e social no espaço.

Assim, visibilidade implica tanto uma dimensão formalespacial como uma dimensão social. No que concerne à dimensão forma-espacial, diversos métodos e métricas do campo visual do espaço têm sido desenvolvidas desde os anos de 1960 e 1970, a saber: [1] Isovista 2D; [2] Isovista 3D; [3] Visibility Graph Analysis (VGA). Além destes, Augmented Visibility Graph Analysis (AVGA) e Real Time 3D Isovist.

[1] Isovista 2D As primeiras noções de isovista foram introduzidas por Tandy (1967) em "The isovist method of landscape survey", mas se tratava de um estudo inserido no campo disciplinar da geografia. Nos anos de 1970, Benedikt (1979) inseriu a noção de isovista nos estudos relacionados à arquitetura, definindo-a como o campo visível a partir de um ponto, considerando as barreiras que o limitam. Tal campo corresponde ao "conjunto de superfícies reais visíveis no espaço" (Benedikt, 1979, p.01), o que, do ponto de vista da geometria, consiste em uma forma poligonal com maior ou menor grau de concavidade. Espaços com áreas similares, mas com configurações poligonais diferentes, geram isovistas com graus de concavidade diferentes. Benedikt (1979) chama essa propriedade de occlusivity (oclusividade) que representa "o quanto os ambientes estão cobrindo parcialmente uns aos outros" (Benedikt, 1979, p.08). Esse atributo é importante porque espaços com maior oclusividade implicam ambientes com mais pontos cegos e baixa intervisibilidade (possibilidade de a partir de um ponto enxergar todos os demais pontos do espaço), aspecto fundamental para a segurança e vigilância social. Contudo, o conceito de isovista proposto por Benedikt (1979) permitia apenas uma análise bidimensional, o que consistia em uma limitação histórica.

[2] Isovista 3D: Em 2009, visando avaliar qualidades espaciais do ambiente urbano relativas à percepção

3 
visual como legibilidade e imageabilidade (Lynch, 2011), Morello e Ratti (2009) desenvolveram novas técnicas de mensuração da percepção do espaço urbano, expandindo o conceito de isovista proposto por Benedikt, incorporando a terceira dimensão. A isovista 3D baseiase no conceito de Spatial Openness (Abertura Espacial) o volume líquido de parte de um sólido definido a partir de um determinado ponto do espaço. Neste caso, considerase o campo de visão tridimensional a partir de um determinado ponto, com intervisibilidade $360^{\circ}$ para todas as direções desse sólido. Isso permite mensurar propriedades fundamentais como área, perímetro, distância radial máxima, mínima, dentre outras, bem como quantificar as chamadas propriedades alongadas que se referem a aspectos como compacidade, concavidade, convexidade, etc.

[3] Visibility Graph Analysis (VGA): A visibilidade começou a ser introduzida na Sintaxe Espacial por Alasdair Turner, Maria Doxa, David O'Sullivan e Alan Penn, em 2001, quando resgataram o conceito de isovista para desenvolver a VGA, uma metodologia de análise de visibilidade através de meios computacionais. Turner et al (2001) acreditavam que, o conceito de isovista poderia ser aprimorado computacionalmente e inserido nas ferramentas de análise espacial, como o DepthmapX. Na VGA, o espaço é decomposto em um grid de pontos submetidos a um método de análise de conexões entre eles - a intervisibilidade -, que implica a visibilidade de dois pontos do espaço entre si. Dessa forma, estabelece-se uma relação de primeira ordem, uma conexão visual, esta relação é quantitativa e mensurável. O espaço, portanto, é plenamente intervisível quando a partir de qualquer ponto seja possível enxergar todos os demais (Turner et al, 2001). Como produto, gera-se um mapa com gradiente de cor (do vermelho ao azul), no qual os espaços mais intervisíveis (mais integrados visualmente) são representados na cor vermelha e os menos intervisíveis (mais segregados) são representados na cor azul.

Além dessas técnicas, destacam-se recentes avanços no campo: (1) Varoudis e Psarra (2014) vêm desenvolvendo isovistas tridimensionais, empregando ferramentas de desenho paramétrico; (3) Varoudis e Penn (2015) têm expandido o conceito de VGA para o que chamam de Augmented Visibility Graph Analysis (AVGA) - uma análise de visibilidade que considera as superfícies transparentes e outros tipos de barreiras que permitem enxergar através; e (3) a Real Time 3D Isovist, em desenvolvimento pelo Computational Planning Group (CPlan), como parte do DeCodingSpaces Toolbox for Grasshopper (Abdulmawla et al, 2017), na BauhausUniversity Weimar.

Pressupõe-se que, esses métodos de análise espacial são essenciais para auxiliar a responder questões sobre como o criminoso enxerga a cidade e tira partido dela para praticar o delito, bem como sobre o modo como a cidade se estrutura (ou se configura espacialmente) para facilitar ou restringir a criminalidade. De modo que, este estudo investiga a aplicabilidade desses métodos de análise espacial (isovistas 2D, 3D e VGA) na avaliação de vulnerabilidade urbana ao crime.

\section{METODOLOGIA}

A metodologia se baseia na correlação entre número de ocorrência de roubos e furtos em segmentos de vias do bairro de Boa Viagem e: (1) medidas extraídas de isovistas 2D (área, perímetro, oclusividade, compacidade); (2) atributos qualitativos de isovistas 3D (mais ou menos cavidades); e (3) valores médios de integração visual (VGA). As isovistas 2D foram elaboradas com o auxílio da ferramenta DeCoding Spaces Toolbox for Grasshopper (Abdulmawla et al, 2017) e as isovistas $3 D$ foram geradas por meio de algoritmos em Rhinoceros 5.0 (McNeel \& Associate, 2015) e Grasshopper 0.9.76.0 (Scott, 2015). Os mapas de VGA foram elaborados no software DepthmapX (Turner, 2001; Varoudis, 2015). Para o estudo, foram escolhidos nove segmentos de via do bairro, uma mostra mínima correspondente a aproximadamente $1 \%$ do número total de roubos e furtos tratados no estudo.

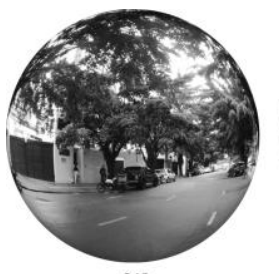

[1]

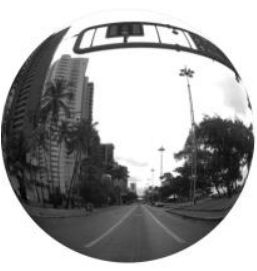

[4]

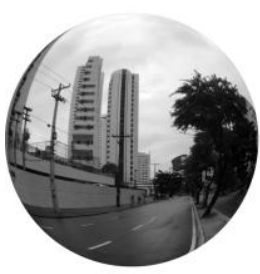

[7]

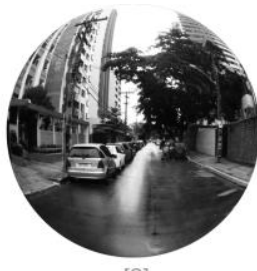

[2]

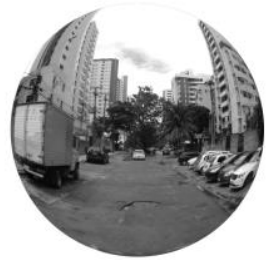

[5]

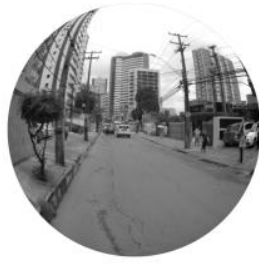

[8]

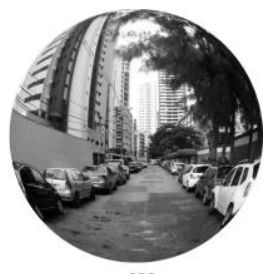

[3]

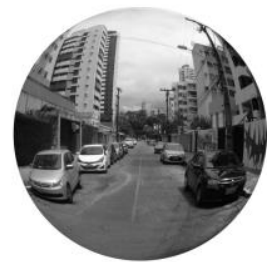

[6]

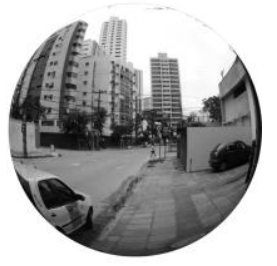

[9]
Figura 2 - Fotos dos segmentos 1 (R. Pe. Carapuceiro), 2 (R. Faustino Porto), 3 (R. Poeta Zezito Neves), 4 (Av. Boa Viagem), 5 (Av. Hélio Falcão), 6 (R. José Moreira Leal), 7 (Av. Eng. Domingos Ferreira), 8 (R. Ribeiro de Brito) e 9 (R. Ernesto de Paula Santos). Fonte: autores.

\section{BASE DE DADOS}

O acesso aos dados de ocorrência de crime dependia da concessão da Secretaria de Defesa Social de Pernambuco (SDS/PE), tendo sido solicitado oficialmente diversas vezes, sem sucesso. Em 10 de setembro de 2015, o ouvidor respondeu à solicitação de número 211/2015-GACE/SDS através do ofício de número 589/2015/GACE/SDS-PE elaborado pelo gerente da GACE/SDS (Gerência de Análise Criminal e Estatística), informando que, os dados georreferenciados são classificados como informação reservada e em alguns casos sigilosa, por isso não seriam disponibilizados. Em função disto, foram solicitados dados de pesquisas recém-realizadas pela Professora Circe Monteiro do Programa de Pós-Graduação em Desenvolvimento Urbano da Universidade Federal de Pernambuco (UFPE), que gentilmente cedeu artigo, contendo espacialização 

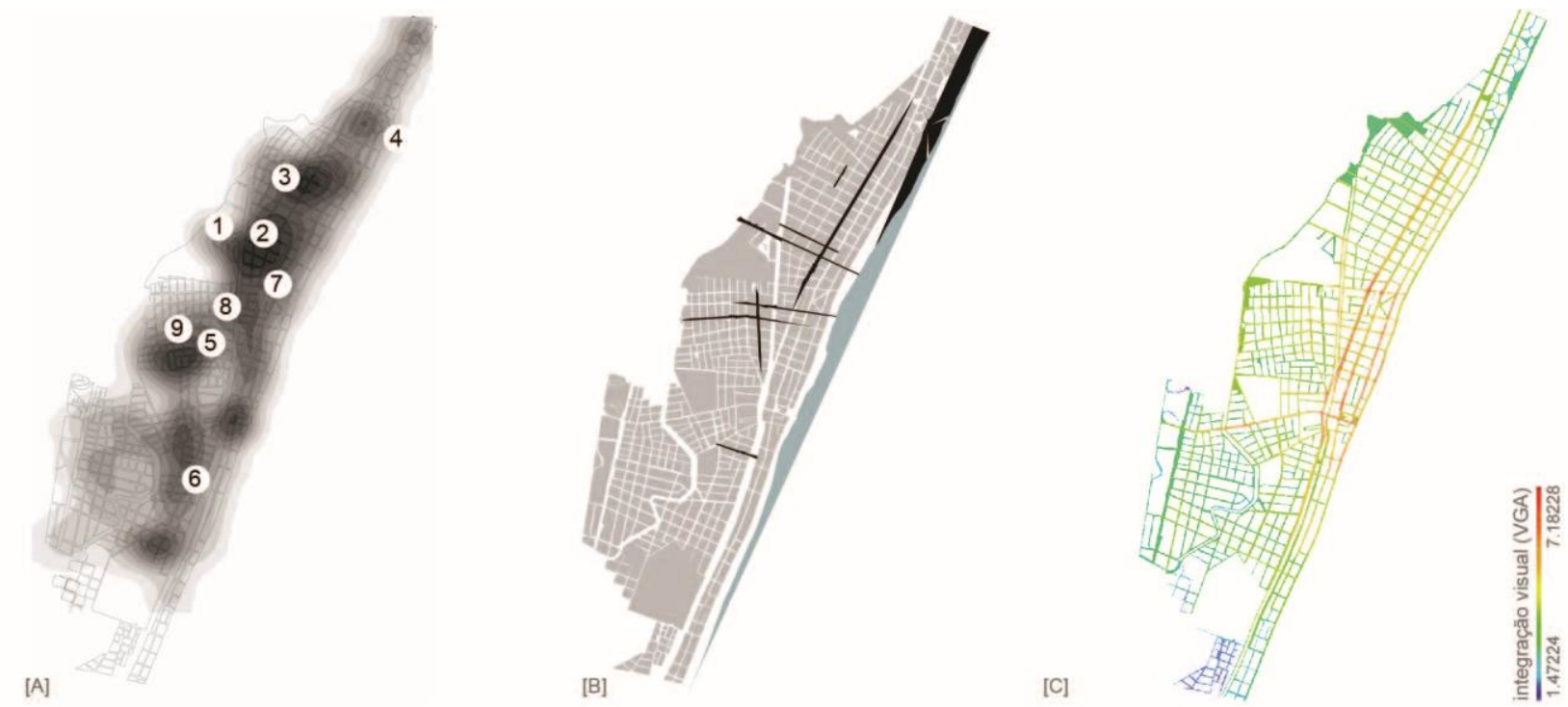

Figura 3 - [A] Mapa Kernel com segmentos identificados, [B] isovistas dos segmentos no teritório, [C] mapa VGA. Fonte: autores.

dos roubos e furtos ocorridos em Boa Viagem, entre os anos de 2010 e 2012 (Monteiro e Cavalcanti, 2015).

Procurou-se, a partir disso, re-sistematizar os dados por meio da confecção de um mapa Kernel que permitisse avaliar a densidade de ocorrência de crimes no bairro de Boa Viagem. O mapa Kernel é uma alternativa para a análise do comportamento de padrões - um método estatístico de estimação de curvas de densidade. Essa peça gráfica facilitou a identificação do padrão de criminalidade urbana no território - regiões com maior e menor densidade de ocorrências.

Em função dessas curvas de densidade apontadas pelo mapa foram escolhidas nove microáreas de análise (segmentos de vias do bairro) como amostras representativas de localidades onde os crimes ocorreram: (1) alta densidade - número elevado de crimes (acima de 10 ocorrências); (2) média densidade - número intermediário de crimes (entre 1 e 10 ocorrências); (3) baixa densidade - pouca ou nenhuma ocorrência.

\section{SEGMENTOS}

Os segmentos 1, 2 e 3 representam espaços de alta densidade de ocorrência de crimes dentre os selecionados. O primeiro está inserido na rua Padre Carapuceiro - um dos principais acessos ao Shopping Recife e onde se encontra o Evolution Shopping Park, um dos maiores condomínios fechados do bairro. O segmento 2 corresponde à rua Faustino Porto, onde se percebe a presença constante de seguranças particulares no espaço público, provavelmente por conta da proximidade com o Colégio Motivo, um dos maiores centros de educação do bairro. $O$ segmento 3 corresponde à Rua Poeta Zezito Neves marcada pela presença de grandes edifícios e pelo baixo número de usuários no espaço público.

O grupo de média densidade de ocorrência é formado pelos segmentos 4,5 e 6 . O segmento 4 está localizado numa das principais vias do bairro, a Avenida Boa Viagem, no trecho correspondente ao $3^{\circ}$ jardim, tendo como característica principal a presença do mar - a praia de Boa Viagem. O segmento 5 (Av. Hélio Falcão) está inserido entre duas vias de tráfego intenso do bairro: Rua Ribeiro de Brito e Rua Ernesto de Paula Santos. O segmento 6, correspondente à Rua José Moreira Leal, é o espaço mais ao sul do bairro.

Os espaços possivelmente mais seguros, com baixa densidade de ocorrência de crimes (ou nenhuma ocorrência), correspondem a trechos da Avenida Engenheiro Domingos Ferreira, Rua Ribeiro de Brito e Rua Ernesto de Paula Santos, respectivamente segmentos 7, 8 e 9. Durante o período analisado, não houve registros de crimes nessas áreas. É importante ressaltar que, os três espaços possuem grande importância viária para o bairro. O segmento 7 corta o território longitudinalmente e as ruas correspondentes aos segmentos 8 e 9 funcionam como um binário que conecta Boa Viagem a bairros vizinhos, como Imbiribeira e Ipsep. A partir desta seleção, foram gerados mapas bidimensionais e modelos tridimensionais, por meio dos quais foi possível gerar isovistas 2D, isovistas 3D e análises gráficas de visibilidade (VGA).

\section{RESULTADOS}

Os resultados são obtidos pela correlação entre duas variáveis quantitativas: o número de ocorrência de crimes e métricas extraídas das isovistas $2 \mathrm{D}$ e da VGA (oclusividade, compacidade, perímetro, área e integração visual (VGA)). As duas variáveis estão correlacionadas se a mudança de uma provoca alterações na outra, seja positiva (relação diretamente proporcional) ou negativa (relação inversamente proporcional). Foram identificadas correlações entre todas as medidas e a ocorrência de crimes, considerando valores normalizados (entre 1 e 5) para facilitar a interpretação das relações, o que pode ser observado nos gráficos de dispersão (ver Gráfico 1).

Tabela 1.1: Resultados extraídos das isovistas e VGA normalizados.

\begin{tabular}{ccccccc}
\hline Seg. & Crimes & Oclusiv. & Perímetro & Área & VGA & Compac. \\
\hline 1 & 14 & 3,08 & 2,72 & 1,27 & 3,32 & 1,15 \\
2 & 14 & 1,57 & 1,61 & 1,07 & 2,22 & 1,84 \\
3 & 14 & 1 & 1 & 1 & 1 & 5 \\
4 & 6 & 4,50 & 5 & 5 & 1,64 & 3,28 \\
5 & 6 & 2,06 & 1,92 & 1,18 & 2,61 & 2,09 \\
6 & 6 & 1,37 & 1,30 & 1,04 & 2,60 & 3,09 \\
7 & 0 & 5 & 4,43 & 1,88 & 5 & 1 \\
8 & 0 & 2,45 & 2,23 & 1,15 & 3,74 & 1,21 \\
9 & 0 & 2,99 & 2,61 & 1,21 & 3,58 & 1,03 \\
\hline Desvio & 1,3764 & 1,3654 & 1,2852 & 1,2026 & 1,3615 \\
\hline \multicolumn{7}{c}{}
\end{tabular}

5 
Tabela 1.2 - Resultados extraídos das isovistas e VGA não normalizados.

\begin{tabular}{ccccccc}
\hline Seg.Crimes & Oclusiv. & Perímetro & Área & VGA & Compac. \\
\hline 1 & 14 & 2220,87 & $2.598,62$ & $19.837,86$ & 3,35 & 0,037 \\
2 & 14 & 751,10 & $1.252,30$ & $7.401,69$ & 3,01 & 0,059 \\
3 & 14 & 185,79 & 499,53 & $3.180,31$ & 2,63 & 0,160 \\
4 & 6 & 3615,78 & $5.378,09$ & $243.017,93$ & 2,84 & 0,105 \\
5 & 6 & 1225,09 & $1.623,43$ & $14.143,25$ & 3,13 & 0,067 \\
6 & 6 & 549,92 & 870,90 & $6.007,67$ & 3,12 & 0,099 \\
7 & 0 & 4098,66 & $4.694,84$ & $56.319,36$ & 3,88 & 0,032 \\
8 & 0 & 1599,31 & $2.005,71$ & $12.494,74$ & 3,48 & 0,039 \\
9 & 0 & 2137,68 & $2.468,83$ & $16.104,42$ & 3,44 & 0,033
\end{tabular}

\section{ISOVISTAS 2D}
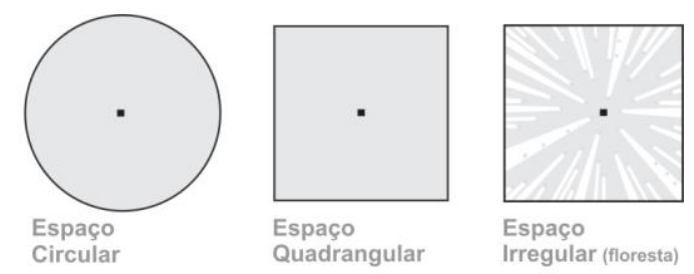

Espaço

Irregular (floresta)

$\begin{array}{ll}\text { Área: } & 400 \\ \text { Perimetro: } & 70,8\end{array}$

Perimetro: $\quad 70,8$

Oclusividade: 0,0

Compacidade: 1,0

Quadrangular
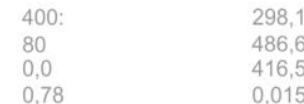

298,1

486,6

416,5

0,015

Figura 4 - Variações em valores de área, perímetro, oclusividade e compacidade em espaços hipotéticos. Fonte: Abdulmawla et al, (2017)

Das isovistas bidimensionais, foram extraídos valores de oclusividade, compacidade, perímetro e área. A oclusividade afere o quanto as barreiras restringem a visão da totalidade do espaço, ou, nas palavras de Benedikt (1979, p.08), 'o quanto os ambientes estão ocultando uns aos outros'. Assim, espaços com alto valor de oclusividade (como os de uma densa floresta), são espaços menos intervisíveis, enquanto espaços com oclusividade baixa, ou próxima a zero, são espaços mais intervisíveis, que propiciam maior domínio do campo visual em sua totalidade (Ver Figura 4).

Correlacionando-se os valores de oclusividade e as ocorrências de crimes, em Boa Viagem, verifica-se uma tendência em ocorrer mais crimes em espaços com valores de oclusividade menor, o que implica dizer que, espaços com baixa oclusividade (ou alta intervisibilidade) são potencialmente mais vulneráveis ao crime. Essa leitura fica mais evidente, quando se observa o gráfico de dispersão (Ver Gráfico 1A). Empiricamente, acredita-se que, criminosos preferem espaços mais oclusivos para atacar suas vítimas, pois nestes há maior possibilidade de esconder-se ou evadir-se, mas os resultados demonstram o oposto - criminosos preferem lugares que Ihes permitam ter maior domínio do campo visual do espaço em sua totalidade.

Em Boa Viagem, em virtude do tecido urbano - uma malha ortogonal ligeiramente deformada, composta predominantemente por ruas e avenidas longas, conformadas entre a praia, os manguezais e o canal -, as isovistas assumem uma forma alongada com áreas e perímetros grandes. A correlação entre área da isovista e a ocorrência de crimes é praticamente perfeita, indicando que há mais roubos e furtos em isovistas com áreas menores (Ver Gráfico 1B e 1D). Cometer crimes em campos visuais menores é, possivelmente, mais uma estratégia utilizada por criminosos. Esse resultado sugere que, preferem ter as vítimas por perto, a distâncias sociais. De acordo com Edward T. Hall (Hall apud Gehl, 2014, p.34), 'podemos ver as pessoas a 100 metros de distância, e se a distância for menor, podemos ver um pouco mais'. Segundo Gehl (2014, p.34) 'reconhecemos uma pessoa à distância de 50 a 70 metros', mesma distância a partir da qual entramos no campo da audição e podemos ouvir gritos. 'A uma distância de 22 a 25 metros, podemos ler corretamente expressões faciais e emoções dominantes'. 'Mas a experiência somente se torna interessante e emocionante a uma distância de menos de dez metros, e de preferência a distâncias ainda menores, quando podemos usar todos os nossos sentidos (Hall apud Gehl, 2014, p.34). Criminosos, portanto, parecem preferir campos visuais menores porque podem usar todos os sentidos deles para cometer o delito.

\section{ISOVISTAS 3D}

As isovistas tridimensionais foram geradas com o auxílio de ferramentas de desenho paramétrico, Rhino e Grasshoper, por meio de algoritmo. A modelagem considerou os edifícios existentes no entorno de cada segmento. Pretendia-se, além disso, explorar atributos como oclusividade tridimensional, volume visível e superfície de cada isovista 3D. Tais análises, todavia, deram-se no campo qualitativo, pois, neste momento da pesquisa, não foi possível extrair métricas por meio da ferramenta DeCodingSpace Toolbox for Grasshoper (Abdulmawla et al, 2017), uma vez que o algoritmo ainda se encontra em desenvolvimento, limitando a investigação.

A análise qualitativa foi realizada com base na observação da existência de mais ou menos cavidades. Essa avaliação foi possível por meio da fabricação digital das isovistas 3D produzidas em acrílico. Verificou-se maior número de cavidades em isovistas correspondentes a ruas onde ocorreram mais crimes, o que leva a concluir que pode haver relação entre oclusividade tridimensional e a ocorrência de crimes. Embora os crimes usualmente ocorram no rés-do-chão, os moradores dos edifícios verticais podem atuar como vigilantes, inibindo a ação de criminoso, particularmente aqueles que residem nos primeiros pavimentos. De acordo com Gehl (2014, p.41), "o contato entre os edifícios e a rua é possível nos primeiros cinco andares. O contato com a cidade rapidamente se dissipa a partir do quinto andar'.

Deste modo, cavidades no campo visual tridimensional gerados por obstáculos visuais, como septos, muros, dentre outros elementos presentes no meio urbano, podem contribuir para aumentar a oclusividade do campo de visão tridimensional dos primeiros pavimentos dos edifícios, tornando o espaço urbano ainda mais vulnerável a ocorrência de crimes. 


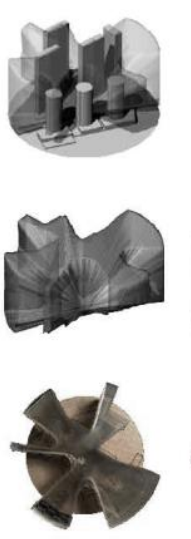

[1]
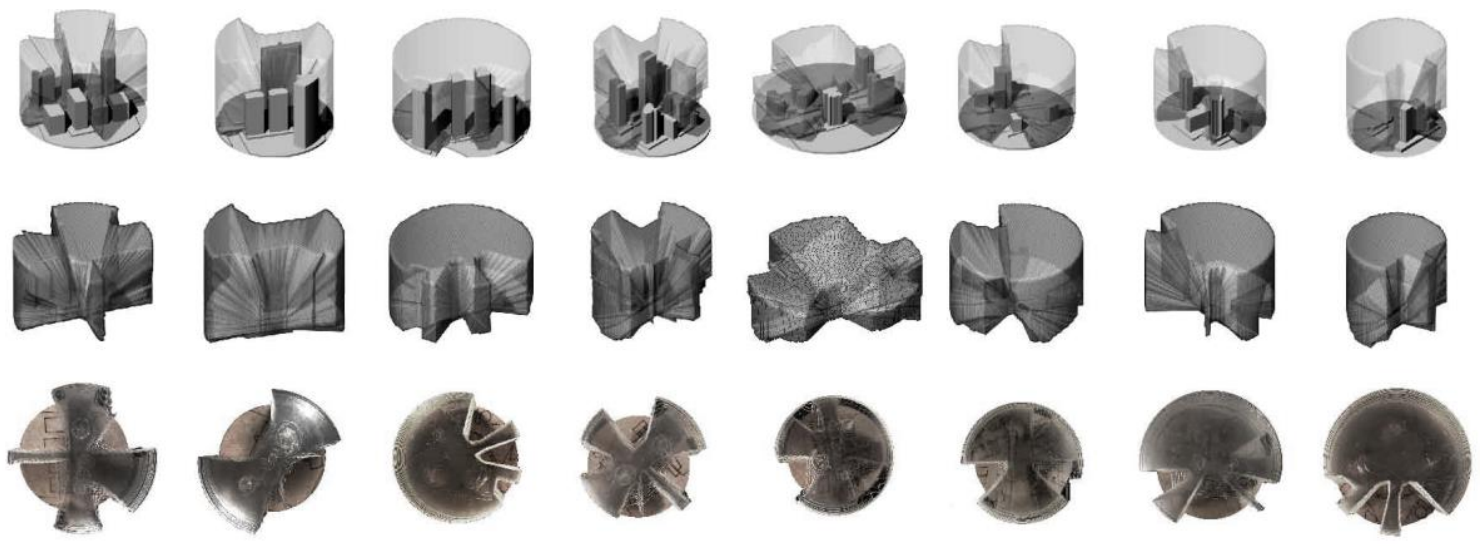

[6]

[7]

[8]

[9]

Figura 5 - Maquete eletrônica das isovistas 3D dos 9 segmentos analisados com o entorno (primeira linha) e sem o entorno (segunda linha) e fotos da maquete física em acrílico (terceira linha). Fonte: autores.

\section{VISIBILITY GRAPH ANALISYS (VGA)}

Para a análise gráfica de visibilidade (VGA), empregou-se o software DepthmapX, que lança uma malha de pontos no sistema de espaços abertos e analisa as relações de intervisibilidade entre os mesmos. Assim, no tecido do bairro de Boa Viagem foi constituída uma malha de 60940 pontos, correspondendo cada um destes a um valor de integração. Os valores de integração variaram entre 7.18228 e 1.47224. Os maiores valores encontrados correspondem a espaços localizados em esquinas de grandes eixos viários, como as avenidas Boa Viagem e Conselheiro Aguiar.

Para calcular a VGA dos nove segmentos de via, foi extraída a média dos valores de integração visual dos pontos contidos no espaço de cada segmento. Os valores médios foram, posteriormente, correlacionados com os números de crimes ocorridos em cada segmento. Observou-se que, há uma tendência de crimes ocorrerem em locais com valores de integração visual mais baixo, o que pode indicar que os criminosos preferem agir ao longo da via a cometer o delito nas esquinas, onde a integração visual é maior (ver Gráfico 1E). Igualmente, preferem espaços mais confinados aos espaços abertos, nos quais a integração visual é maior.

Em geral, grids urbanos nos quais a proporção de espaços abertos é maior que a proporção de espaços fechados, como no plano piloto de Brasília, o valor de integração visual geral é maior. Em contraposição, grids morfologicamente mais próximos de cidades tradicionais, em que a proporção de espaços fechados é maior que a proporção de espaços abertos, o valor de integração visual é menor. Contudo, em ambos os casos, as esquinas terão sempre valores maiores de VGA do que ao longo da rua.

Assim, é possível que o criminoso até prefira se posicionar nas esquinas antes da ação criminosa, para ter maior domínio visual das vítimas, mas preferem efetuar o roubo ou o furto em trechos visualmente menos integrado, sem que possa ser visto por mais pessoas e sem dar tempo de reação à vítima de, por exemplo, escapar para outras direções, aspecto facilitado pelas esquinas.

\section{DISCUSSÃO}

Certamente, a ocorrência de crimes no meio urbano não é determinada por um único atributo morfológico do espaço, como a visibilidade, nem mesmo por todas as variáveis da forma urbana, pois envolve aspectos que estão para além deste estudo. A criminalidade urbana é um dos problemas desencadeados principalmente pela fragilidade da função social da cidade, especialmente nos grandes centros. Problemas sociais como desemprego e desprovimento de serviços públicos básicos assistenciais como saúde e educação, dentre outras debilidades, favorecem a proliferação da marginalidade. Consequentemente, a sociedade procura se proteger dentro do espaço privado e se utiliza de tecnologias e soluções arquitetônicas de proteção (câmeras, muros, gradis, etc.), visando, quase sempre, ao encarceramento.

Contudo, é evidente que, a constituição de barreiras visuais tem implicações significativas para o campo visível e social do espaço, pois altera sua configuração, suas propriedades mórficas e, consequentemente, as relações sociais a serem estabelecidas, pois altera o modo como o percebemos.

De acordo com, Eduard T. Hall (2005), "o espaço formado pela visão é importante no estabelecimento das relações humanas e socioespaciais, sendo responsável pela apropriação do mesmo. A natureza do espaço visual é muito mais complexa que a natureza do espaço auditivo, por exemplo, pois uma barreira de som a distância causa menos impedimentos do que uma barreira visual". Ainda de acordo com Hall (2005, p.79-88), "a quantidade de informações transmitidas pela visão é muito maior, quando comparada a qualquer outro sentido. Por meio da visão, o ser humano é capaz de desempenhar diversas
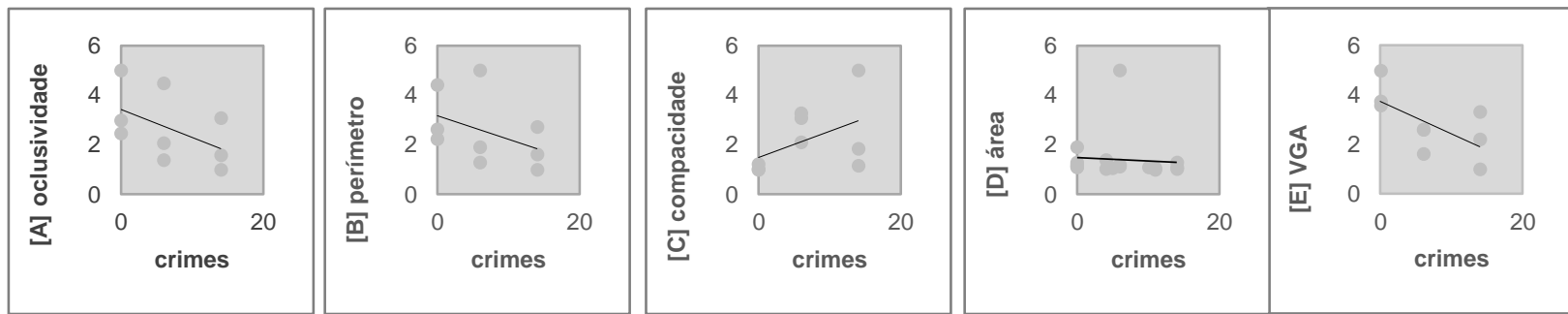

Gráfico 1 - Gráficos de correlação entre crimes e oclusividade [A], perímetro [B], compacidade [C], área [D] e VGA [E]. 
funções como identificar alimentos, amigos e o estado físico de materiais a certa distância. Também, pode orientar-se dentro de um espaço e evitar obstáculos e perigos, criar ferramentas, cuidar de si mesmo e dos outros, tecer avaliações e colher informações. No espaço visual está tudo aquilo que enxergamos, isto é, o conjunto de cores em movimentações constantes e, no mundo visual, residem as percepções do que representa aquilo que está sendo enxergado: perigo, conforto, segurança, etc. Neste contexto, a visão periférica também desponta como essencial para a segurança dos seres humanos, pois ainda que não tenha acuidade visual perfeita nessas regiões é possível identificar perigos iminentes ou ainda elementos secundários à visão.

Deste modo, é bastante provável que, criminosos se utilizem de todos os recursos sensoriais possibilitados pela visão para cometer o delito e a configuração do espaço pode contribuir para facilitar sua atuação ou restringi-la. É imprescindível o desenvolvimento de mais investigações neste campo. Relações ainda mais complexas entre os atributos morfológicos do espaço, percepção e psicologia ambiental devem ser investigadas.

\section{CONCLUSÃO}

Buscou-se identificar relações entre visibilidade e criminalidade, bem como avaliar a aplicabilidade de isovistas $2 \mathrm{D}, 3 \mathrm{D}$ e análises gráficas de visibilidade (VGA) na avaliação de vulnerabilidade urbana ao crime, visando contribuir com os estudos locais e internacionais.

Embora o número de amostras seja reduzido, os resultados sugerem que, criminosos preferem cometer delitos em locais com baixa oclusividade, escolhem espaços ao longo das ruas em detrimento espaços localizados nas esquinas, optam por espaços mais compactos, com menor área e perímetro de campo visual, em detrimento de grandes espaços abertos. Para facilitar o delito atuam em espaços onde circulam pessoas e selecionam locais menos oclusivos e compactos para que possam ter maior domínio visual de suas vítimas. Esses resultados demonstram que, de fato, isovistas e análises gráficas de visibilidade têm grande potencial para serem aplicadas na aferição da vulnerabilidade espacial ao crime, bem como na proposição de soluções de mitigação de ocorrência de crime no meio urbano.

Por fim, um futuro aprofundamento desta pesquisa é a ampliação do número de amostras e extensão do estudo a outros bairros da cidade, além da incorporação das métricas referentes às isovistas 3D (o que foi limitado nesta etapa). Além disto, um possível desdobramento é empregar instrumentos analíticos mais avançados como Augmented Visibility Graph Analysis (AVGA). Nos últimos anos, estudos no campo da sintaxe espacial têm apontado limitações da análise de visibilidade, como o fato de a isovista possuir certa rigidez ao considerar as bordas simplesmente como barreiras visuais e físicas opacas, negligenciando elementos como transparências, mobiliários, paredes a meia altura, elementos reflexivos, etc., que podem alterar a acessibilidade, mas não a visibilidade. Varoudis e Penn (2015) argumentam que as análises sintáticas sofrem também uma simplificação associada a essas limitações e, para superá-las, vêm aprimorando a Augmented Visibility Graph Analysis
(AVGA) que considera diferenças de relações entre os pontos no espaço, como transparências, mobiliário, barreiras à meia altura, entre outros. Essas técnicas, se aplicadas nos estudos de relações entre visibilidade e criminalidade, poderão elucidar um número maior de questões.

\section{REFERÊNCIAS}

ABDULMAWLA, Abdulmalik et al. DeCodingSpaces Toolbox: for Grasshopper. Version 2017.01. [S.I.: s.n.], 2017. Retrieved from: http://decodingspaces-toolbox.org.

BENEDIKT, M L. To take hold of space: isovists and isovists fields. 1979.

BRASIL, Decreto-lei no 2.848, Código Penal Brasileiro, de 7 e dezembro de 1940.

GEHL, Jan. Cidade para pessoas. São Paulo: Perspectiva, 2014. $280 \mathrm{p}$.

HALL, Edward T.. A Dimensão Oculta. São Paulo: Martins Fontes, 2005.

HILLIER, Bill. Can streets be made safe? 2004. Retrieved from: http://www.futurecommunities.net/files/images/CAN_STREET S_BE_MADE_SAFE_1_.pdf.

HILLIER, Bill; SAHBAZ, Ozlem. An evidence based approach to crime and urban design: Or, can we have vitality, sustainability and security all at once?. 2008. Retrieved from: http://spacesyntax.com/wp-content/uploads/2011/11/HillierSahbaz_An-evidence-based-approach_010408.pdf

JACOBS, Jane. Morte e Vida de Grandes Cidades. 3. ed. São Paulo: Wmf Martins Fontes, 2011.

LYNCH, Kevin. A Imagem da Cidade. São Paulo: Wmf Martins Fontes, 2011.

MICHAELIS. Visibilidade. In: MICHAELIS. Visibilidade. São Paulo: Melhoramentos Ltda, 2016.

MONTEIRO, Circe Maria Gama; CAVALCANTI, Rafaella dos Santos. Spatial profile of urban segments: Assessing place vulnerability to crime. In: INTERNATIONAL SPACE SYNTAX SYMPOSIUM, 10., 2015, Londres. Proceedings... . Londres: Sss, 2015. p. 1 - 17. Retrieved from: http://www.sss10.bartlett.ucl.ac.uk/wpcontent/uploads/2015/07/SSS10_Proceedings_136.pdf

MORELLO, Eugenio; RATTI, Carlo. A Digital Image of the City: 3-D isovists and a tribute to Kevin Lynch. 2009. Retrieved from: http://dspace.mit.edu/handle/1721.1/55992.

NEWMAN, Oscar. Creating Defensible Space. Washington: Rutgers University, 1996. $126 \mathrm{p}$.

NEWMAN, Oscar. Defensible Space: Crime Prevention Through Urban Design. New York: Macmillan Publishing, 1972. 264 p.

PERNAMBUCO, Diario de. Recife está entre as 40 cidades mais violentas do mundo. Diário de Pernambuco. Recife, p. 1-1. abr. 2016.

Tandy C R. The Isovist Method Of Landscape Survey. In: METHODS OS LANDSCAPE ANALYSIS, 1., 1967

TURNER, Alasdair et al. From isovists to visibility graphs: a methodology for the analysis of architectural space. 2001.

VAROUDIS, Tasos; PENN, Alan. Visibility, accessibility and beyond: Next generation visibility graph analysis. In: INTERNATIONAL SPACE SYNTAX SYMPOSIUM, 10. 2015, Londres. Proceedings... . Londres: Ucl, 2015. p. 1 - 13. Retrieved from: <http://www.sss10.bartlett.ucl.ac.uk/wpcontent/uploads/2015/07/SSS10_Proceedings_152.pdf>.

VAROUDIS, Tasos; PSARRA, Sophia. Beyond two dimensions: Architecture through three-dimensional visibility graph $\begin{array}{lll}\text { analysis. } & 2014 . & \text { Retrieved from: } \\ \text { http://joss.bartlett.ucl.ac.uk/journal/index.php/joss/article/view }\end{array}$ http://joss.bartlett.ucl.ac.uk/journal/index.php/joss/article/view
/200 $/ 200$

ZELINKA, Al; BRENNAN, Dean. Safescape: Creating Safer, More Livable Communities Through Planning \& Design. New York: Planners Pr, 2000. 